\title{
Hybrid catalytic membranes: tunable and versatile materials for fine chemistry applications
}

\author{
Yingying $\mathrm{Gu}^{\mathrm{a}}$, Clélia Emin ${ }^{\mathrm{a}}$, Jean-Christophe Remigy ${ }^{\mathrm{a}}$, Isabelle Favier ${ }^{\mathrm{b}}$, Montserrat \\ Gómez $^{\mathrm{b}}$, Richard D. Noble ${ }^{\mathrm{c}}$, Douglas L. Gin ${ }^{\mathrm{c}}$, Jorge Macanás ${ }^{\mathrm{d}}$, Berta Domènech ${ }^{\mathrm{e}}$, Jean- \\ François Lahitte ${ }^{\text {a }} 1$ \\ ${ }^{a}$ Université de Toulouse, INPT, UPS, CNRS, Laboratoire de Génie Chimique, France \\ ${ }^{b}$ Laboratoire Hétérochimie Fondamentale et Appliquée, UMR CNRS 5069, Université de Toulouse 3 - Paul Sabatier, France \\ ${ }^{c}$ Department of Chemical \& Biological Engineering, University of Colorado, Boulder, United States \\ ${ }^{d}$ Department of Chemical Engineering, Universitat Politècnica de Catalunya, UPC, Spain \\ ${ }^{e}$ Chemistry Department, Universitat Autònoma de Barcelona, Spain
}

\begin{abstract}
The aim of this work is the synthesis of polymer-stabilized Pd nanoparticles inside a functionalized polymeric porous membrane and to test them in metal-catalyzed organic reactions. A polymeric membrane support (Polyethersulfone) was functionalized with an ionogen polymer capable of retaining metallic nanoparticles (MNP) or MNP precursors using a UV photo-grafting method. Membranes were also obtained by casting sulfonated polyethersulfone (Cardotype). The reactions were carried out by filtering solutions containing the reactants leading to full conversions within seconds. The amount of PdNP included in the materials and the catalytic activity were influenced by the properties of the grafted network.

Copyright (C) 2014 Elsevier Ltd. All rights reserved.

Keywords: membrane; nanoparticle; catalysis; functionalization; poly(ionic liquid); Suzuki-Miyaura cross-coupling
\end{abstract}

\section{Introduction}

The raise of nanotechnology has provided the use of transition Metal Nanoparticles (MNP) as effective and selective catalysts, which can be used inside polymeric matrices. Consequently, the development of hybrid metal-polymer nanocomposite catalytic membranes has notably increased in recent years. The simple incorporation of ex-situ generated MNP into a polymeric matrix is made possible by immersion of the membrane in a colloidal solution [1]. However, adsorption is sometimes not efficient enough to retain the catalyst. Alternatively, Intermatrix Synthesis (IMS) is a technique based on the immobilization of MNP precursors (metal ions or complexes) into polymeric matrices followed by their chemical or electrochemical reduction. This technique provides the production of desired hybrid metal-polymer nanocomposite materials, which have already demonstrated their catalytic and bactericidal properties [2].

With the aim to avoid the leaching and improve the performance of MNP, IMS has to be applied to polymers bearing ionogen or chelating groups that bind to MNP precursors. For this issue, a polymeric membrane support (Polyethersulfone on flat sheet or hollow fiber form) was functionalized with an ionogen polymer capable of retaining PdNP or PdNP precursors (i.e. poly(acrylic acid), poly(styrene sulfonate) or poly(ionic liquid) (imidazolium-based derivatives) PIL using an UV photo-grafting method. PdNP were then generated inside the polymeric matrix by chemical reduction of salts (in situ synthesis) or captured by the polymeric matrix after synthesis in solution (ex situ synthesis) [3]. The catalytic performance of the asprepared PdNP catalytic membranes was evaluated using various reactions such as chemical reduction of nitrophenol and Suzuki-Miyaura cross-coupling between 4-nitroiodobenzene and phenylboronic acid. The reactions were carried out by filtering solutions containing the reactants leading to full conversions within seconds compared to hours under batch conditions. An overview of the most important results of our joint international collaboration is presented here.

\footnotetext{
* Corresponding author. Tel.: +33561556829

E-mail address: lahitte@chimie.ups-tlse.fr
} 


\section{Results and discussion}

\subsection{Modification of the membrane surface}

We applied two different methods to obtain ionic functionalities in the membrane materials. One of them concerns the surface modification of an existing membrane; the other one is modifying the membrane using charged polyelectrolytes. The first approach permits the use of a preexisting, commercially available membrane or with a well-documented fabrication process [4-5]. The second one requires the development and control of a new modification process of a porous material, which can be difficult or even impossible to obtain by the classical phase inversion process due to polyelectrolytes solubility in water.

Photo-polymerization allows the polymeric membrane surface modification by the action of UV-light irradiation upon the chromophoric groups present in the polymeric unit of the material. Radical formation, induced by the UV irradiation, initiates a radical polymerization leading to the formation of polymeric chains. When the radicals are formed at the membrane surface, the polymeric chain grows from the surface. This "grafting from" technique produces polymeric chains covalently linked to the membrane surface in a permanent way [6]. The homopolymerization of monomers in the feed solution can be avoided by the choice of an appropriate wavelength of UV radiation. Actually, the UV radiation is preferentially absorbed by the membrane surface rather than by monomer present in the feed solution. Free polymers in solution (it means no linked polymers to the membrane) have to be eliminated by several washing steps with water or a good solvent of the grafted polymer non solvent of the membrane. Use of a cross linker permits to interconnect these chains to form a cross-linked network. The polymeric graft exhibits a gel-like structure in these conditions. The thickness of the grafted layer is function of several parameters such as monomer and cross linker concentration, viscosity of the solution, and irradiation time [3].

When the polymer absorbs UV irradiation, UV penetration is shallow and the graft polymeric layer is localized at the surface, predominantly out of the membrane. On the contrary, when UV irradiation penetrates deep inside the membrane, grafted polymer is localized mainly inside the membrane as an interpenetrated network. This UV absorption depends on the absorption spectra of the polymer and the Beer Lambert law but also on the membrane structure. Open porous structure allows the UV penetration far inside the membrane whereas dense structure limits the UV penetration to few micrometers.

For this work, we investigated the grafting of three different monomers, acrylic acid (AA), sodium styrene sulfonate (NaSS), and the polymerizable ionic liquid (imidazolium-based derivative such as 1,2dimethyl-3-propylimidazolium /(trifluoromethylsulfonyl)imide) (PIL). All monomers have been successfully grafted at the membrane surface.

The structure analysis of the grafted layer indicates different organizations depending on the nature of the monomer. Since acrylic acid is a highly reactive monomer, it polymerizes before penetration into the membrane. Thus, the polymeric layer is clearly localized out of the membrane, as observed in the Scanning electron microscopy (SEM) analysis shown in Fig. 1.

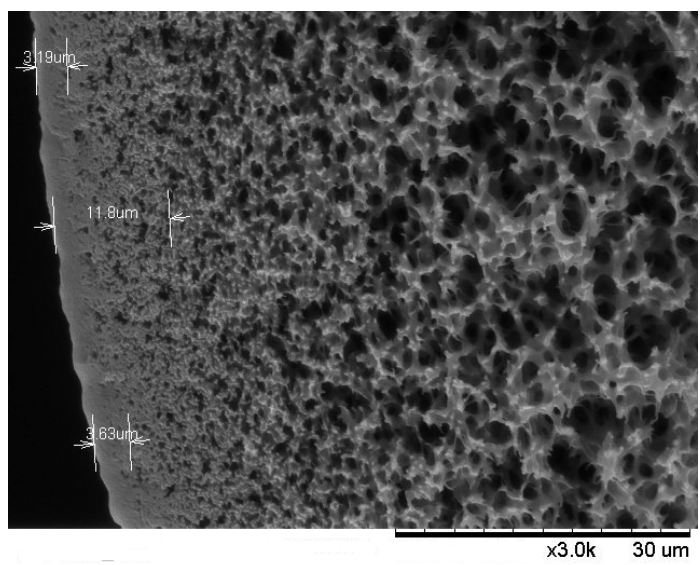

Fig. 1: SEM image of PAA (poly(acrylic acid)) grafted on MicroPES® hollow fiber cross section

Under these conditions, NaSS was less reactive than AA and diffuses into the membrane before polymerizing. In this condition, an interpenetrated network (IPN) is observed instead of a clear dense layer. The same tendency was observed with the PIL. 
Alternatively and with the aim to compare with the grafting technique with a different procedure, the constituting polymer was chemically modified by sulfonation before elaborating the membrane. The functionalization was achieved by a direct sulfonation of Polyethersulfone Cardo type (PES-C) with concentrated sulfuric acid [7]. Flat sheet membranes were obtained for PES-C with a sulfonation degree lower than $30 \%$. It is noteworthy that higher sulfonation extent was not possible due to the increase of the hydrophilicity and the water solubility of the sulfonated polymer.

\subsection{Metallic nanoparticles incorporation in the membrane}

Pd nanoparticles in the membrane were synthesized by the IMS methodology. Transmission electronic microscopy (TEM) was used to observe the localization of the PdNP in the membrane, the average diameter and dispersion of the PdNP, and the presence of possible aggregates (Fig.2). For all types of grafted layers, we observed that NP were largely localized in the graft layer and practically absent outside it. No significant aggregation was observed. The diameter of the NP ranged from 2 to $5 \mathrm{~nm}$ in the polyelectrolyte grafted layers and from 6 to $13 \mathrm{~nm}$ in the sulfonated polymer membrane. The difference of NP diameter can be explained by the different structure of these two types of materials. In the grafted layer, the medium is a polymeric network where nanoparticles stabilized by electrostatic and steric means, are entrapped. It is clearly observed that the structure of the polyelectrolyte has an effect on the amount of Pd that can be incorporated into the membrane. The highest metal loading (expressed by the ratio meq $\mathrm{Pd}^{2+} / \mathrm{meq}$ polymer charge) , $80 \mathrm{meq} / \mathrm{m}^{2}$ of $\mathrm{Pd}^{2+}$, was obtained for PAA in form of a polymeric network, swelled by the solvent (water), and localized outside the membrane. In this case, charges are well accessible for the ion exchange. For PSS and PIL, the grafted polymer was mainly localized inside the membrane in a form of an interpenetrated network with lower accessibility than in the case of PAA, giving an outside gel network. For sulfonated PES-C, an important part of ionic groups was localized inside the bulk material without being accessible to the $\mathrm{Pd}^{2+}$ solution for an anionic exchange with the counter ion. Thus, $\mathrm{Pd}^{2+}\left(0.4 \mathrm{meq} / \mathrm{m}^{2}\right)$ loading, expressed in milliequivalent (meq) per surface of membrane $\left(\mathrm{m}^{2}\right)$, was less important than in the former case. The highest efficiency was observed with PAA swelling network attaining with incorporation 8 times superior than expected for a stoichiometric ionic exchange. Nevertheless, network pores could probably act as a pool of $\mathrm{Pd}^{2+}$ solution, feeding the reaction. In the case of PIL, ion exchange is retarded by the poor mobility of the counter ion $\left(\mathrm{NTf}_{2}^{-}\right)$. Results are summarized in Table 1.

Table 1: Pd content and loading efficiency for the different prepared nanocomposite films.

\begin{tabular}{lllllll}
\hline $\begin{array}{l}\text { Nature of the } \\
\text { polyelectrolyte }\end{array}$ & $\begin{array}{l}\text { Type } \\
\text { grafted } \\
\text { layer }\end{array}$ & $\begin{array}{l}\text { of } \\
\text { polymer } \\
\text { charge/m }\end{array}$ & of & meq of $\mathrm{Pd}^{2+} / \mathrm{m}^{2}$ & $\begin{array}{l}\mathrm{meq} \mathrm{Pd}^{2+} / \mathrm{meq} \\
\text { polymer charge }\end{array}$ & $\begin{array}{l}\mathrm{NP} \\
\text { diameter } \\
(\mathrm{nm}) \\
\mathrm{nm}\end{array}$ \\
\hline PAA & $\begin{array}{l}\text { Partially } \\
\text { cross-linked }\end{array}$ & & & mean \\
& 5.8 & 12.1 & 2.1 & 5 \\
PAA & Gel & 10.0 & 80.0 & 8.0 & 5 \\
PSS & IPN & 0.5 & 1.56 & 2.9 & 5 \\
PIL & IPN & 20.8 & 12 & 0.6 & 2 \\
Sulfonated & PES- & $\begin{array}{l}\text { Bulk } \\
\text { modified }\end{array}$ & 2.0 & 0.4 & 0.2 & 13 \\
\hline
\end{tabular}

${ }^{a}$ PAA $=$ Poly (Acrylic Acid); PSS = sodium Poly(Styrene Sulfonate); PIL = Poly Ionic Liquid ${ }^{\mathrm{b}}$ Based on TEM observations

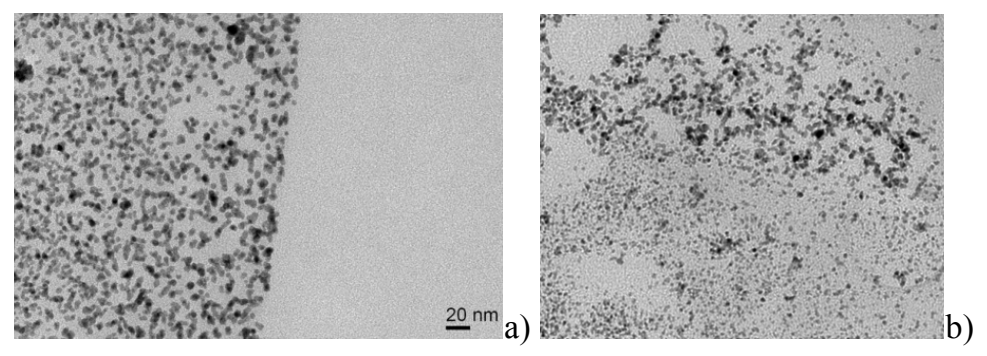




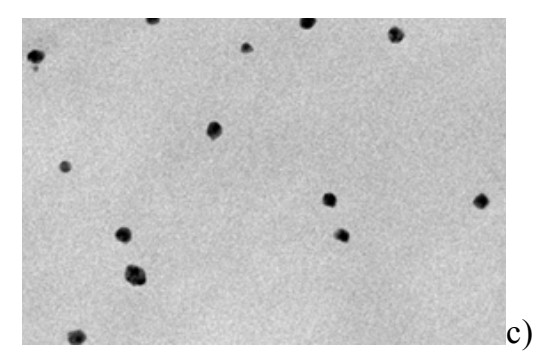

Fig.2: TEM images of PdNP in the membranes: a) PAA; b) PIL; and c) sulfonated PES-C (same scale for all pictures)

\subsection{Catalytic behavior}

The catalytic performance of the PdNP catalytic membranes was evaluated in two processes, chemical reduction of 4-nitrophenol (eq 1) and the Suzuki-Miyaura cross-coupling between 4-nitroiodobenzene and phenyl boronic acid (eq 2) [8]. These benchmark catalytic reactions were carried out by filtering solutions containing the reactants through the as-prepared membranes.

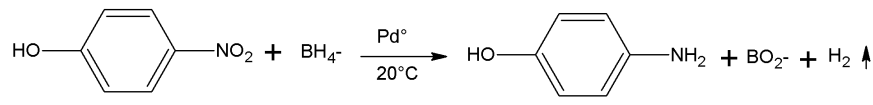

The reduction of 4-nitrophenol (4-NP) in 4-aminophenol (4-AP) took place using $\mathrm{NaBH}_{4}$ in water as reducing agent (conditions: $0.72 \mathrm{mmol}$ of 4-NP; $14.38 \mathrm{mmol}$ of $\mathrm{NaBH}_{4}$; solution flux: $20 \mathrm{Lh}^{-1} \mathrm{~m}^{-2} ; 20^{\circ} \mathrm{C}$ )

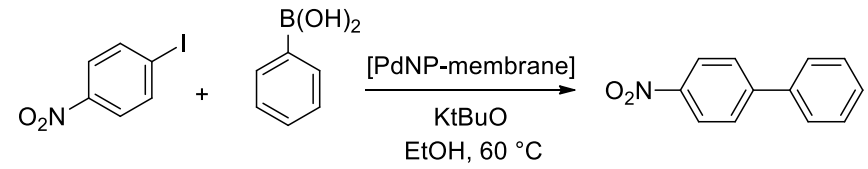

The Suzuki-Miyaura cross-coupling reaction involved 1-iodo-4-nitrobenzene and phenyl boronic acid as reagents and potassium tert-butoxide as base (conditions: [1-iodo-4-nitrobenzene] = $32 \mathrm{mmol} / \mathrm{L}$; molar ratio: 1-iodo-4-nitrobenzene/phenyl boronic $\mathrm{acid} / \mathrm{KtBuO} / \mathrm{Pd}=1 / 1.2 / 2.5 / 0.007$; solution flux: $40 \mathrm{Lh}^{-1} \mathrm{~m}^{-2} ; 60{ }^{\circ} \mathrm{C}$ )

The catalytic membranes with PdNP included in a grafted layer were much more efficient than under batch conditions or using colloidal nanoparticle systems, in terms of activity and chemoselectivity. Conversions higher than $99 \%$ were obtained with a single filtration step corresponding to a contact time with PdNP of only few seconds (3-10 s), in contrast to 1 hour (4-nitrophenol reduction) or 6 hours (SuzukiMiyaura cross coupling) required to reach the same conversion in batch reactor conditions. Membranes were reusable more than 10 times without significant loss of their catalytic activity, giving metal-free organic products. These high conversion and efficiency of the process are due to the important PdNP concentration in the grafted layer (500 to 1000 more concentrated than in colloidal solution of PdNP with the same amount of $\mathrm{Pd}$ ) together with the convective flow which allows the feeding of the PdNP and thus enhancing the kinetic of the processes. In the case of the sulfonated polymer membrane, lower catalytic efficiency of PdNP was observed (conversion of 15\%), in agreement with both the lesser PdNP concentration and the PdNP dispersion in all the membrane pore surface. But total conversion was accomplished in a multipass mode configuration (permeate being reinjected to the feed solution).

\section{Conclusions}

Polymeric membranes can easily be functionalized by polyelectrolytes and polyelectrolyte networks are efficient materials to synthesize the host material and to stabilize metallic nanoparticles of small diameter preventing aggregation. By this means, the resulting material, a hybrid metallic/polymeric nanocomposites material, became multifunctional exhibiting high reactivity in Pd-catalyzed reactions and permitted process intensification by separation of products and reactants during the process. The amount of PdNP included in the materials and the catalytic activity were influenced by the properties of the grafted network, from branched to gel-like structure. 


\section{Acknowledgements}

The authors gratefully acknowledge the Ministry of Education and Research French, Paul Sabatier University and the National Center of Scientific Research (CNRS) ) as well as ACC1O for VALTEC0902-0058 grant within the "Programa Operatiu de Catalunya" (FEDER).

\section{References}

[1] L. Ouyang, D.M. Dotzauer, S.R. Hogg, J. Macanás, J.F. Lahitte, M.L. Bruening, Catal. Today 156 (2010) 100-106.

[2] P. Ruiz, M. Muñoz, J. Macanás, C. Turta, D. Prodius, D.N. Muraviev, Dalton Trans. 39 (2010) 1751-1757.

[3] C.Emin, J-C. Remigy, J-F. Lahitte, J. Membr. Sci., 455 (2014) 55-63.

[4] A. Akbari, S. Desclaux, J.C. Rouch, J.C. Remigy, J. Membr. Sci. 297(2007) 243-252.

[5] T. Goma-Bilongo, A. Akbari, M.J. Clifton, J.C. Remigy, J. Membr. Sci. 278 (2006) 308-317.

[6] H.Yamagishi , J.V. Crivello, G.Belfort, J.Membr. Sci. 105 (1995) 237-247.

[7] B.Domènech , M. Muñoz, D.N. Muraviev, J. Macanás, Nanoscale Research Letters 6 (2011)406-411.

[8] For a selected contribution of PdNP applied in catalysis, see: F. Chahdoura, C. Pradel, M. Gómez, Adv. Synth. Catal. 355 (2013) 3648-3660. 